\title{
Merging Carrot Linkage Groups based on Conserved Dominant AFLP Markers in $\mathbf{F}_{2}$ Populations
}

\author{
Carlos A. F. Santos ${ }^{1}$ \\ Embrapa, Brazilian Agricultural Research Corporation, Semi-Arido, Caixa Postal 23, 56302-970, \\ Petrolina, PE. Brazil \\ Philipp W. Simon ${ }^{2}$ \\ U.S. Department of Agriculture-Agricultural Research Service, Vegetable Crops Research Unit, \\ Department of Horticulture, 1575 Linden Drive, University of Wisconsin, Madison, WI 53706
}

\begin{abstract}
AdDitional INDEX WORDS. amplified fragment length polymorphism, coupling linkage maps, Daucus carota, genome coverage, molecular markers

AвSTRACT. Markers were placed on linkage groups, ordered, and merged for two unrelated $\mathrm{F}_{2}$ populations of carrot (Daucus carota L.). Included were 277 and 242 dominant Amplified fragment-length polymorphism (AFLP) markers and 10 and eight codominant markers assigned to the nine linkage groups of Brasilia $\times$ HCM and B493 x QAL F 2 populations, respectively. The merged linkage groups were based on two codominant markers and 28 conserved dominant AFLP markers (based upon sequence and size) shared by both populations. The average marker spacing was 4.8 to $5.5 \mathrm{cM}$ in the four parental coupling phase maps. The average marker spacing in the six merged linkage groups was $3.75 \mathrm{cM}$ with maximum gaps among linkage groups ranging from 8.0 to $19.8 \mathrm{cM}$. Gaps of a similar size were observed with the linkage coupling phase maps of the parents, indicating that linkage group integration did not double the bias which comes with repulsion phase mapping. Three out of nine linkage groups of carrot were not merged due to the absence of common markers. The six merged linkage groups incorporated similar numbers of AFLP fragments from the four parents, further indicating no significant increase in bias expected with repulsion phase linkage. While other studies have merged linkage maps with shared AFLPs of similar size, this is the first report to use shared AFLPs with highly conserved sequence to merge linkage maps in carrot. The genome coverage in this study is suitable to apply quantitative trait locus analysis and to construct a cross-validated consensus map of carrot, which is an important step toward an integrated map of carrot.
\end{abstract}

Carrot is a diploid $(2 \mathrm{n}=2 \mathrm{x}=18)$, outcrossing species, with small chromosomes of relatively uniform length demarked by three secondary constrictions (Sharma and Bhattacharyya, 1954). The carrot genome size is $473 \mathrm{Mbp}$ or $0.98 \mathrm{pg}$ of DNA per haploid genome (1C) (Arumuganathan and Earle, 1991) which is approximately four times the genome size of Arabidopsis L., the same size as rice (Oryza sativa $\mathrm{L}$.), one half the size of tomato (Lycopersicum esculentum L.), one fifth of corn (Zea mays L.) and one twentieth the size of onion (Allium cepa L.). Vivek and Simon (1999) estimated the carrot genome to be $900 \mathrm{cM}$ in length which corresponds to $520 \mathrm{~kb}$ per cM.

Unlike the high density linkage maps for crops, such as soybean (Keim et al., 1997), maize (Vuylsteke et al., 1999), barley (Castiglioni et al., 1998), sugar beet (Schafer-Pregl et al., 1999), Pinus (Remington et al., 1999) and Eucalyptus (Marques et al., 1998), the published genetic maps of carrot are low-density, clearly unsaturated, and with minimal coverage of the carrot genome since only around 350 points have been mapped (Schulz et al., 1994; Vivek and Simon, 1999; Westphal and Wricke, 1989, 1991, 1997). Development of codominant markers rarely has been attempted and a limited number of PCR-based markers have been reported in the carrot literature (Boiteux, 2000; Bradeen and Simon, 1998, Niemann et al., 1997, Vivek and Simon, 1999). Mapping of $F_{2}$ populations with primarily dominant markers (Schulz et al., 1994; Vivek and Simon, 1999) has been used to increase the numbers of markers on carrot maps but this marker class has contributed little toward the development of reliable marker order within linkage

Received for publication 2 June 2003. Accepted for publication 28 Oct. 2003. 'Corresponding author; e-mail psimon@wisc.edu. groups, since different $\mathrm{F}_{2}$ populations were used with different studies and no attempt to develop a consensus map was made, marker density was not increased as more markers were added to independent maps.

To establish a system of joining carrot maps from diverse genetic backgrounds, two linkage maps derived from two unrelated crosses including one between wild white (Daucus carota var. carota) $\mathrm{x}$ cultivated orange (Daucus carota ssp. sativus) carrots and the other between cultivated orange $\mathrm{x}$ cultivated dark orange carrots. Maps consisted mostly of amplified fragment length polymorphisms (AFLPs) and a few randomly amplified polymorphic DNAs (RAPDs), sequence characterized amplified regions (SCARs) and microsatellites, and they were merged based on two codominant markers they shared and on 28 sequenceconserved dominant AFLP markers. AFLPs generated from the same primer pairs and of the same size have been used to join maps (e.g., Alonso-Blanco et al., 1998) and for this study we sequenced putative shared bands and used those with high sequence homology $(>91 \%)$ since we have determined that these sequence-conserved bands are much more likely to share map location in carrot (Santos and Simon, 2002a). The goal of the present study was to merge both maps as part of a larger goal of constructing a cross-validated consensus map of carrot.

\section{Material and Methods}

Plant material, DNA eXtraction, MOlecular marker aPPROACHES AND IDENTIFICATION OF CONSERVED AFLP FRAGMENTS. Mapping populations were obtained by self-pollinating single $\mathrm{F}_{1}$ hybrid plants which originated from a cross between B493 x QAL 
(inbred line $\mathbf{x}$ wild carrot; cross 1 , ) and Brasilia x HCM (openpollinated Brazilan cultivar $\mathrm{x}$ high carotene population; cross 2). Leaf samples from 183 and $160 \mathrm{~F}_{2}$ plants, from cross 1 and 2 respectively, were harvested and prepared for DNA extraction.

Total genomic DNA was isolated from freeze-dried leaves as described by Boiteux et al., (1999). DNA concentration was determined with a TKO 100 Mini Fluorometer (Hoefer Scientific Instruments, San FranciscoCalif.) using bis-benzimidazole dye (Hoechst 33258) and an estimate of total DNA concentration (mg of DNA/g) was obtained by comparing with a calf thymus DNA standard. The size, purity, and integrity of total genomic DNA was also determined on $0.8 \%$ agarose gels.

The AFLP method was performed essentially as described in Vos et al., (1995), with minor adaptations for carrot DNA introduced by Vivek and Simon (1999). Genomic DNA was separately double digested using the restriction endonuclease combinations EcoR1/MseI and PstI/MseI. Each polymorphic AFLP fragment was identified by 1) six letters corresponding to the primer combination, followed by 2) the estimated number of nucleotides of the DNA fragment, and 3) a letter indicating the parental origin of the fragment: $\mathrm{B}, \mathrm{H}, 4$ and $\mathrm{Q}$, respectively for Brasilia, HCM, B493 and QAL. Polymorphic AFLP markers in both populations, which were digested with the same endonuclease enzyme, amplified with the same primer combination and shared the same position in a polyacrylamide gel, were selected for elution and re-amplification and further sequencing (Santos and Simon, 2002a). Those proved to be identical in nucleotide sequence were used as anchor to merge both linkage maps in order to identify conserved AFLP fragments.

LINKAGE ANALYSES, HETEROGENEITY TEST, AND GROUP MERGING. Linkage analyses were performed with the software MapMaker version 3.0 (Lander et al., 1987) and with the software JoinMap version 2.0 (Stam and van Ooijen, 1995). AFLP fragments, scored as 0 or 1 for absence or presence, were codified to symbols required by MapMaker that are the same symbols recognized by JoinMap. Where the parental allelic phase was unknown, markers were double-scored. The phase was deduced by association with phase-known linkages (Vivek and Simon, 1999) and based on the warning message issued by the module JMREC in the JoinMap software, when the recombination fraction exceeded 0.6 (Stam and van Ooijen, 1995).

Segregation distortion tests were performed with JoinMap. Linkage groups were assigned primarily with the JoinMap software using LOD scores ranging from 3.0 to 6.5 and maximum recombination fraction of 0.5 . The options used in the JoinMap module JMMAP were: LOD score of 2.0, recombination threshold of 0.49 , jump Chi-square threshold of 6.0 and triplet jump Chi-square threshold of 6.0. The recombination fraction frequencies were converted to Kosambi centiMorgans (cM) (Kosambi, 1944).

Only the maps generated with the JoinMap software were considered for further analysis since merging of maps is possible only in JoinMap. Heterogeneity testing between the common markers in both populations was performed with the JMHET module available in the JoinMap. Linkage group merging was performed with a dataset that included markers in coupling and repulsion phases. Maps were drawn with DrawMap software (van Ooijen, 1994) kindly provided by the developer.

\section{Results and Discussion}

Generation of dominant polymorphic AFLPs. A total of 421 and 317 polymorphic AFLP fragments were scored in the
Brasilia x HCM and B493 x QAL $\mathrm{F}_{2}$ populations, respectively. The chi-square $p$ values were $\leq 0.02$ for $32 \%$ and $23 \%$ of the AFLP fragments in Brasilia x HCM and B493 x QAL $F_{2}$ populations, respectively, and these fragments were not included in the linkage grouping analysis. Marques et al., (1998) also used chi-square tests with reliable screening of markers and $\approx 15 \%$ of the bands displayed skewed segregation ratios of total AFLP fragments initially scored from further consideration in a linkage study of different Eucalyptus species. The AFLP fragment size ranged from 54 to 700 nucleotides in the two carrot $F_{2}$ populations, which is similar to the size of the AFLP products in other reports (Marques et al., 1998, Remington et al., 1999; Vivek and Simon, 1999).

EcoRI/MseI and $P$ stI/MseI enzymes pairs revealed 12.0 and 17.8 polymorphic fragments per primer combination, respectively, in the Brasilia $\times \mathrm{HCM} \mathrm{F}_{2}$, and 11.3 and 11.9 polymorphic fragments per primer combination, respectively, in the B493 x QAL $F_{2}$. A higher percentage of $P s t \mathrm{I} / \mathrm{Mse} \mathrm{I}$ fragments $(71.5 \%$ in the Brasilia $\mathrm{X}$ HCM population and $87.0 \%$ in the B $493 \times$ QAL population) than EcoRI/MseI AFLP fragments (64.9\% in the Brasilia $x$ HCM population and $70.8 \%$ in the B493 $\times$ QAL population) had chi-square values $>0.02$. In this study, more well-defined and easier to score polymorphic AFLP fragments were usually observed with PstI primers than with EcoRI primers. Vuylsteke et al., (1999) found a higher percentage of polymorphism among $P s t \mathrm{I} / M s e \mathrm{I}$ primers than among $E c o \mathrm{RI} / M$ seI primers in a large study of two populations of maize. However, Young et al., (1999) reported that fewer restriction fragments were generated by Pst-AFLP than with EcoAFLP in a soybean mapping study. PstI and EcoRI are well known as methylation-sensitive and nonmethylation-sensitive restriction enzymes, respectively (Keim et al., 1999). It is reasonable to assume that repetitive DNA in the heterochromatic regions leads to more imprecise AFLP fragments, due to comigration of similar amplified products. Scoring would therefore not be as precise as with single copy regions amplified from euchromatin regions. According to Young et al., (1999), a mapping strategy combining methylationsensitive and methylation-insensitive enzymes, $P$ stI and $E c o$ RI, with the AFLP technique should provide more complete map coverage and allow for the targeting of specific regions based on genomic methylation patterns. PstI-derived RFLP clones have been used in mapping studies to increase the frequency of markers in genetically active euchromatic regions (Burr et al., 1988).

GENERATION OF CODOMINANT POLYMORPHIC AFLPS, MICROSATELLITES AND SCARs. Loci producing two cosegregating fragments in repulsion phase and amplified with the same primers have been considered as codominant in RAPD marker studies (Grattapaglia and Sederoff, 1994; Krutovskii et al., 1998) and also in AFLP analysis (Vivek and Simon, 1999). In this study, if two AFLP fragments in repulsion were amplified by the same primer combination and they presented a recombination fraction of $\leq 5.0$ $\mathrm{cM}$ based on individual linkage analysis of a primer combination with the MapMaker software, then they were treated as codominant markers in our linkage analysis. A true codominant marker, scored by chance as dominant marker in AFLP analysis, has a recombination fraction of $\approx 5.0 \mathrm{cM}$ and LOD score around 4.0 in a population size of 150 individuals. Converted AFLP marker pairs never occurred in the double absent configuration, thus meeting the requirements of codominant markers. The chi-square tests for codominant AFLP ranged from $6.8 \%$ to $89.3 \%$ in the two $\mathrm{F}_{2}$ populations. It is necessary to point out that this method is different from the trans-dominant linked markers approach (Plomion et al., 1996), where a maximum-likelihood estimator is 
applied to estimate the recombination fraction of markers linked in repulsion phase. Eight and four AFLP markers were converted to codominant markers in the Brasilia $\times$ HCM and B493 x QAL $\mathrm{F}_{2}$ populations, respectively. This result corresponds to $1.91 \%$ and $1.24 \%$, respectively, of all AFLP fragments scored. Vivek and Simon (1999) reported a higher conversion to codominant AFLP, 3\%, based on autoradiogram analysis.

Most of published microsatellites (Niemann et al., 1997) and PCR-based codominant markers (Boiteux 2000; Bradeen and Simon 1998) were evaluated in both populations of this study. Only one microsatellite published by Niemann et al., (1997) yielded codominant information in both populations. Three PCR products of the RAPD OP-K9C primer, which were scored as codominant markers by Vivek and Simon (1999), were cloned and sequenced. Primers were redesigned as SCAR in order to give unambiguous and easy to score codominant information. The microsatellite sequences (5'ATAATAGGGCGTTTCTTGTTCTT3' and 5'CGGGTAGGCTTAATGTTCTGT3', identified in routine sequencing of carrot PCR products, provided codominant information in $2.5 \%$ agarose gels for the B493 x QAL F 2 population only.

Among fifteen SCARs based on polymorphic conserved sequences and closely linked AFLP fragments between Brasilia x HCM and B493 x QAL $\mathrm{F}_{2}$ populations, only one provided codominant information. Five other SCARs were designed based on amplicon sequencing information of Brasília-1252 x B6274 $\mathrm{F}_{2}$ population, in an attempt to merge linkage maps, but all failed to provide codominant information. Considering the time consuming and low efficiency of the SCAR approach to convert dominant information into codominant information (Paran and Michelmore, 1993), this approach was not pursued further. The overall approach to develop codominant information yielded 8 markers in the B493 $\times$ QAL $F_{2}$ population and 10 markers in the Brasilia $x \mathrm{HCM} \mathrm{F}_{2}$ population.

LINKAGE ANALYSIS: SEGREGATION DISTORTION, LINKAGE GROUPS AND MARKER ORDER. Molecular markers with chi-square probability values $<0.02$ were dropped from the linkage analysis. The decision to use this value for this study was based on grouping patterns at different $p$ value levels: $0.001,0.01,0.02$ and 0.05 . These are the cutoff chi-square $p$ values used to drop markers due to segregation distortion. Nine similar linkage groups were observed at $p$

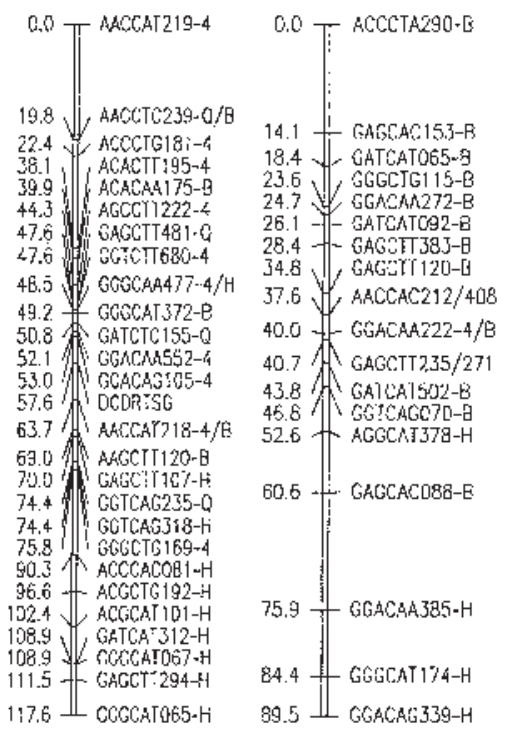

value of 0.02 and 0.05 which is important for carrot where $\mathrm{n}=\mathrm{x}$ $=9$. More than nine groups were observed when $p$ values of 0.01 and 0.001 were included in the linkage analysis. Among those markers with $p$ values between 0.02 and 0.05 , nineteen $(6.6 \%)$ and twenty $(8.0 \%)$ of the markers effectively used to assign linkage groups showed segregation distortion in the Brasilia x HCM and $\mathrm{B} 493 \times \mathrm{QAL} \mathrm{F}_{2}$ populations, respectively. The $p$ value for all codominant markers was $>0.05$ in both populations. The linkage mapping literature has reported mapping analysis with $p$ values $>0.01$ (Krutovskii et al., 1998; Marques et al., 1998; Remington et al.1999; Vivek and Simon, 1999) and >0.05 (Vuylsteke et al., 1999). In this study, mapping markers with segregation distortion giving values lower than 0.01 led to clustering of many markers in one linkage group and only a few markers in other linkage groups, which results in skewed distributions of markers among linkage groups and larger numbers of linkage groups than expected for the carrot genome $(n=x=9)$. According to Liu (1997), care should be taken in interpreting the linkage hypothesis tests when segregation distortion exists because the statistically significant linkages are likely false positives. The same author also noted that most of the available computer software for linkage analysis and genomic map construction cannot analyze markers showing distortion from Mendelian inheritance. Segregation analysis has also been associated with directional clustering of distorted markers and excess or underrepresentation of alleles from one parent (Stam and van Ooijen, 1995; Vuylsteke et al., 1999).

The overall marker data sets were consistently assigned to nine linkage groups at LOD scores ranging from 3.5 to 5.0 in both $\mathrm{F}_{2}$ populations. MapMaker and JoinMap determined exactly the same number of groups and assigned the same markers for a given group using the same LOD score, indicating that algorithms used by these two software packages led to identical results. Vuylsteke et al., (1999) commented that the LOD score used by JoinMap is not affected by the segregation distortion, and that normal LOD score used by MapMaker can lead to spurious linkage of markers with segregation distortion, which was not observed in the present study. Linkage grouping performed only with markers linked in coupling phase showed the same nine linkage groups at LOD scores ranging from 3.0 to $7.5,3.0$ to $5.5,3.5$ to 5.0, and 2.5 to 8.5 in the Brasilia-, HCM-, B493-, and QAL-coupling $\mathrm{F}_{2}$

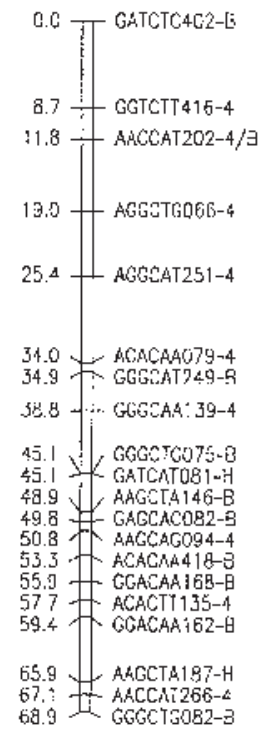

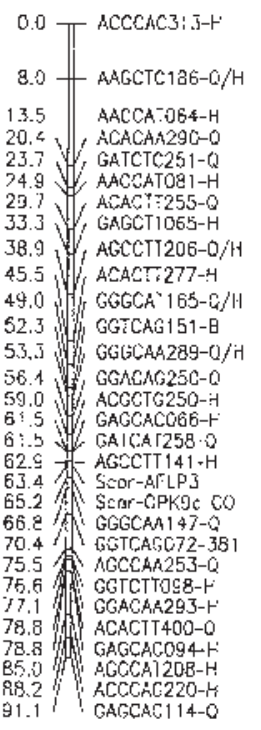

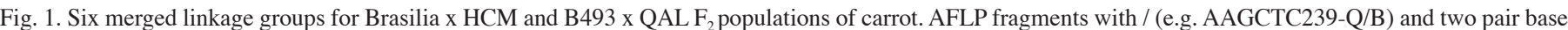
sizes (e.g. GAGCTT235/271) represent a conserved AFLP fragment between the two populations and a codominant AFLP, respectively. 
populations, respectively. According to Knapp et al., (1995), the recombination fraction estimation with dominant markers in repulsion phase in the $\mathrm{F}_{2}$ is seriously biased due to the low frequency of the double recessive class in the progeny. However, the same authors stated that the estimation of locus grouping seldom poses a problem because a locus can be assigned to a group on the basis of $\left(\mathrm{k}^{2}-\mathrm{k}\right) / 2$ tests. Groupings obtained with LOD thresholds in the range of 4.0 to 7.0 are best (Stam and van Ooijen, 1995).
Ordering poses much more of a problem than grouping mixedphase dominant markers (Knapp et al., 1995) because the recombination frequency estimates are frequently biased, as Mather (1936) showed 67 years ago. Using the inverse of the variance of recombination fraction obtained with the method of maximum likelihood, Mather (1936) showed that repulsion-linked dominant markers in $\mathrm{F}_{2}$ populations are most efficient with the absence of linkage. With the exceptions of linkage groups 1, 2 and 6 and link-

Table 1. Heterogeneity test $(\mathrm{H})$ for recombination fraction $(\theta)$ per linkage group among common molecular markers used to merge linkage groups between the Brasilia (Bsb) $\times$ HCM and B493 $\times$ QAL $\mathrm{F}_{2}$ carrot populations (P).

\begin{tabular}{|c|c|c|c|c|c|c|}
\hline \multirow[b]{2}{*}{ Pair } & \multirow[b]{2}{*}{$\mathrm{F}_{2}$} & \multirow[b]{2}{*}{$\theta$} & \multirow[b]{2}{*}{ LOD } & \multicolumn{2}{|c|}{ Chi-square } & \multirow{2}{*}{$\begin{array}{c}\text { Linkage } \\
\text { group }\end{array}$} \\
\hline & & & & $\mathrm{P}$ & $\mathrm{H}$ & \\
\hline \multirow[t]{2}{*}{ AACCAT218-4/B x AGGCTA108-4/H } & Bsb $\times$ HCM & 0.3033 & 1.42 & 3.70 & & LG1 \\
\hline & B493 x QAL & 0.1340 & 14.47 & 1.69 & $5.39^{*}$ & LG1 \\
\hline \multirow[t]{2}{*}{ AACCAT218-4/B x GAGCAC444-4/H } & Bsb $\times$ HCM & 0.2005 & 9.02 & 0.07 & & LG1 \\
\hline & B493 x QAL & 0.2401 & 2.36 & 0.19 & $0.26^{\mathrm{Ns}}$ & LG1 \\
\hline \multirow[t]{2}{*}{ AACCAT218-4/B x DCDRTSG } & Bsb $\times$ HCM & 0.0658 & 23.19 & 1.26 & & LG1 \\
\hline & B493 x QAL & 0.1230 & 16.49 & 1.05 & $2.30^{\mathrm{Ns}}$ & LG1 \\
\hline \multirow{2}{*}{ AACCAT218-4/B x GATCTC181-4/H } & Bsb $\times$ HCM & 0.1937 & 2.47 & 0.17 & & LG1 \\
\hline & B493 x QAL & 0.1561 & 12.14 & 0.04 & $0.22^{\mathrm{Ns}}$ & LG1 \\
\hline \multirow{2}{*}{ AGGCTA108-4/H x DCDRTSG } & Bsb $\times$ HCM & 0.3194 & 2.01 & 7.43 & & LG1 \\
\hline & B493 x QAL & 0.1044 & 18.73 & 5.70 & $13.12^{* *}$ & LG1 \\
\hline \multirow{2}{*}{ AGGCTA108-4/H x GAGCAC444-4/H } & Bsb $\times$ HCM & 0.0928 & 19.58 & 1.21 & & LG1 \\
\hline & B493 x QAL & 0.1704 & 8.57 & 1.43 & $2.64^{\mathrm{Ns}}$ & LG1 \\
\hline \multirow{2}{*}{ AGGCTA108-4/H x GATCTC181-4/H } & Bsb $\times$ HCM & 0.0722 & 16.43 & 2.24 & & LG1 \\
\hline & B493 x QAL & 0.0133 & 32.85 & 2.67 & $4.91^{*}$ & LG1 \\
\hline \multirow[t]{2}{*}{ GAGCAC444-4/H x GATCTC181-4/H } & Bsb $\times$ HCM & 0.0658 & 23.15 & 0.87 & & LG1 \\
\hline & B493 x QAL & 0.1229 & 11.44 & 1.04 & $1.91^{\mathrm{Ns}}$ & LG1 \\
\hline \multirow[t]{2}{*}{ GAGCAC444-4/H x DCDRTSG } & Bsb $\times$ HCM & 0.1870 & 9.79 & 1.95 & & LG1 \\
\hline & B493 x QAL & 0.3247 & 2.01 & 2.49 & $4.44^{*}$ & LG1 \\
\hline \multirow[t]{2}{*}{ DCDRTSG x GATCTC181-4/H } & Bsb $\times$ HCM & 0.2902 & 2.77 & 5.19 & & LG1 \\
\hline & B493 x QAL & 0.1102 & 17.07 & 4.11 & $9.30^{* *}$ & LG1 \\
\hline \multirow[t]{2}{*}{ GGACAA222-4/B x GGGCAT282-4/H } & Bsb $\times$ HCM & 0.2551 & 1.95 & 4.45 & & LG2 \\
\hline & B493 x QAL & 0.0805 & 18.82 & 2.14 & $6.59^{*}$ & LG2 \\
\hline \multirow[t]{2}{*}{ GGACAA222-4/B x AAGCAG075-4/H } & Bsb $\times$ HCM & 0.3509 & 0.85 & 0.80 & & LG2 \\
\hline & B493 x QAL & 0.2561 & 4.58 & 0.43 & $1.23^{\mathrm{Ns}}$ & LG2 \\
\hline \multirow[t]{2}{*}{ GGGCAT282-4/H x GGACAG272-4/H } & Bsb $\times$ HCM & 0.3339 & 1.05 & 0.08 & & LG2 \\
\hline & B493 x QAL & 0.3676 & 1.09 & 0.05 & $0.13^{\mathrm{Ns}}$ & LG2 \\
\hline \multirow[t]{2}{*}{ GGGCAT282-4/H x AAGCAG075-4/H } & Bsb $\times$ HCM & 0.2824 & 3.23 & 0.15 & & LG2 \\
\hline & B493 x QAL & 0.2435 & 4.85 & 0.15 & $0.31^{\mathrm{Ns}}$ & LG2 \\
\hline \multirow{2}{*}{ AAGCAG075-4/H x GGACAG272-4/H } & Bsb $\times$ HCM & 0.2334 & 4.64 & 1.51 & & LG2 \\
\hline & B493 x QAL & 0.1304 & 12.07 & 1.39 & $2.91^{\mathrm{Ns}}$ & LG2 \\
\hline GGACAG259-4/B x ACACAA142-4/H & Bsb $\times$ HCM & 0.2477 & 1.68 & 0.39 & & LG3 \\
\hline & B493 x QAL & 0.1834 & 8.08 & 0.15 & $0.54^{\mathrm{Ns}}$ & LG3 \\
\hline GGACAG259-4/B x AGCCAA136-Q/H & Bsb $\times$ HCM & 0.3835 & 0.53 & 0.84 & & LG3 \\
\hline & B493 x QAL & 0.2584 & 2.56 & 0.83 & $1.67^{\mathrm{Ns}}$ & LG3 \\
\hline ACACAA142-4/H x AGCCAA136-Q/H & Bsb $\times$ HCM & 0.3984 & 0.67 & 0.98 & & LG3 \\
\hline & B493 x QAL & 0.2564 & 2.54 & 1.66 & 2.64 ss & LG3 \\
\hline AACCAT100-4/H x AAGCAG097-4/B & Bsb $\times$ HCM & 0.2256 & 1.97 & 0.86 & & LG4 \\
\hline & B493 x QAL & 0.1434 & 15.03 & 0.22 & $1.09^{\mathrm{Ns}}$ & LG4 \\
\hline AACCAT100-4/H x GGGCAT119-Q/H & Bsb $\times$ HCM & 0.3308 & 1.83 & 0.01 & & LG4 \\
\hline & B493 x QAL & 0.3224 & 2.13 & 0.01 & $0.01^{\mathrm{Ns}}$ & LG4 \\
\hline AAGCAG097-4/B x GGGCAT119-Q/H & Bsb $\times$ HCM & 0.3200 & 1.20 & 0.27 & & LG4 \\
\hline & B493 x QAL & 0.2527 & 2.80 & 0.24 & $0.51^{\mathrm{Ns}}$ & LG4 \\
\hline AACCAT202-4/B x ACACAA106-Q/B & Bsb x HCM & 0.0979 & 15.43 & 0.23 & & LG5 \\
\hline & B493 x QAL & 0.0000 & 4.82 & 2.80 & $3.04^{\mathrm{Ns}}$ & LG5 \\
\hline АACCAT202-4/B x ACACTT153-4/H & Bsb $\times$ HCM & 0.2567 & 2.01 & 0.01 & & LG5 \\
\hline & B493 x QAL & 0.2465 & 5.22 & 0.00 & $0.01^{\mathrm{Ns}}$ & LG5 \\
\hline AACCAT202-4/B x GGGCAA229-4/H & Bsb $\times$ HCM & 0.4136 & 0.34 & 0.01 & & LG5 \\
\hline & B493 x QAL & 0.4228 & 0.35 & 0.01 & $0.01^{\mathrm{Ns}}$ & LG5 \\
\hline
\end{tabular}


age groups 6 and 7, respectively, in Brasilia x HCM and B493 x QAL populations, the coupling + repulsion map was the same as that of one parent (Santos, 2001). The linkage group exceptions had at least two codominant markers in common and mapping to the same group, thus reducing the bias due to the presence of repulsion phases. Jiang and Zeng (1997) concluded that $12 \%$ of dominant and recessive markers are in the correct order with a marker density of $5 \mathrm{cM}$, increasing to $41.0 \%$ for a marker density of $20 \mathrm{cM}$.
A similar shortage of codominant markers to order the linkage groups with mixed linkage phase was also reported by Vivek and Simon (1999) in the most comprehensive mapping effort in carrot to date. Among the 11 linkage groups reported by them, three linkage groups had two codominant markers and five linkage groups had one codominant marker, which had led to a simple coupling linkage map in eight out of eleven groups reported.

LINKAGE GROUP MERGING OF BRASILIA X HCM AND B493 X QAL

Table 1.Continued.

\begin{tabular}{|c|c|c|c|c|c|c|}
\hline \multirow[b]{2}{*}{ Pair } & \multirow[b]{2}{*}{$\mathrm{F}_{2}$} & \multirow[b]{2}{*}{$\theta$} & \multirow[b]{2}{*}{ LOD } & \multicolumn{2}{|c|}{ Chi-square } & \multirow{2}{*}{$\begin{array}{c}\text { Linkage } \\
\text { group }\end{array}$} \\
\hline & & & & $\mathrm{P}$ & $\mathrm{H}$ & \\
\hline \multirow[t]{2}{*}{ ACACAA106-Q/B x ACACTT153-4/H } & Bsb x HCM & 0.2442 & 2.34 & 0.02 & & LG5 \\
\hline & B493 x QAL & 0.2206 & 2.33 & 0.03 & $0.05^{\mathrm{ss}}$ & LG5 \\
\hline \multirow[t]{2}{*}{ ACACAA106-Q/B x GGGCAA229-4/H } & Bsb $\times$ HCM & 0.3395 & 0.99 & 0.74 & & LG5 \\
\hline & B493 x QAL & 0.4418 & 0.21 & 0.44 & $1.18^{\mathrm{Ns}}$ & LG5 \\
\hline \multirow[t]{2}{*}{ ACACTT153-4/H x GGGCAA229-4/H } & Bsb $\times$ HCM & 0.2869 & 3.56 & 0.81 & & LG5 \\
\hline & B493 x QAL & 0.2033 & 7.23 & 0.86 & $1.66^{\mathrm{Ns}}$ & LG5 \\
\hline \multirow[t]{2}{*}{ AGCCTT206-Q/H x GATCTC386-4/B } & Bsb $\times$ HCM & 0.3503 & 0.70 & 1.10 & & LG6 \\
\hline & B493 x QAL & 0.1834 & 3.10 & 1.35 & $2.45^{\text {Ns }}$ & LG6 \\
\hline \multirow[t]{2}{*}{ AGCCTT206-Q/H x Scar-AFLP3 } & Bsb x HCM & 0.2191 & 5.37 & 0.14 & & LG6 \\
\hline & B493 x QAL & 0.1915 & 11.62 & 0.08 & $0.22^{\mathrm{Ns}}$ & LG6 \\
\hline \multirow[t]{2}{*}{ AGCCTT206-Q/H x ACACAA319-4/H } & Bsb x HCM & 0.1808 & 8.08 & 0.59 & & LG6 \\
\hline & B493 x QAL & 0.0000 & 5.21 & 5.62 & $6.21^{*}$ & LG6 \\
\hline \multirow{2}{*}{ AGCCTT206-Q/H x GGGCAT165-Q/H } & Bsb $\times$ HCM & 0.1420 & 10.27 & 0.20 & & LG6 \\
\hline & B493 x QAL & 0.1157 & 20.08 & 0.13 & $0.32^{\mathrm{Ns}}$ & LG6 \\
\hline \multirow[t]{2}{*}{ AGCCTT206-Q/H x GGTCTT350-4/H } & Bsb $\times$ HCM & 0.1524 & 9.16 & 0.40 & & LG6 \\
\hline & B493 x QAL & 0.0000 & 4.52 & 4.11 & $4.51^{*}$ & LG6 \\
\hline \multirow[t]{2}{*}{ AGCCTT206-Q/H x GGGCAA289-Q/H } & Bsb $\times$ HCM & 0.1637 & 7.87 & 0.35 & & LG6 \\
\hline & B493 x QAL & 0.1260 & 18.61 & 0.2 & $0.55^{\mathrm{Ns}}$ & LG6 \\
\hline \multirow[t]{2}{*}{ GATCTC386-4/B x Scar-AFLP3 } & Bsb $\times$ HCM & 0.0552 & 24.71 & 0.01 & & LG6 \\
\hline & B493 x QAL & 0.0605 & 22.45 & 0.01 & $0.03^{\mathrm{ss}}$ & LG6 \\
\hline \multirow[t]{2}{*}{ GATCTC386-4/B x ACACAA319-4/H } & Bsb $\times$ HCM & 0.1539 & 4.21 & 0.90 & & LG6 \\
\hline & B493 x QAL & 0.2445 & 4.69 & 0.38 & $1.27 \mathrm{Ns}$ & LG6 \\
\hline \multirow[t]{2}{*}{ GATCTC386-4/B x GGGCAT165-Q/H } & Bsb $\times$ HCM & 0.2374 & 2.35 & 0.42 & & LG6 \\
\hline & B493 x QAL & 0.1519 & 4.39 & 0.46 & $0.88^{\mathrm{ss}}$ & LG6 \\
\hline \multirow[t]{2}{*}{ GATCTC386-4/B x GGTCTT350-4/H } & Bsb $\times$ HCM & 0.3220 & 1.02 & 3.51 & & LG6 \\
\hline & B493 x QAL & 0.1389 & 11.45 & 1.75 & $5.25^{*}$ & LG6 \\
\hline \multirow[t]{2}{*}{ GATCTC386-4/B x GGGCAA289-Q/H } & Bsb $\times$ HCM & 0.2729 & 1.45 & 0.69 & & LG6 \\
\hline & B493 x QAL & 0.1553 & 4.04 & 0.69 & $1.38^{\mathrm{Ns}}$ & LG6 \\
\hline \multirow[t]{2}{*}{ Scar-AFLP3 x ACACAA319-4/H } & Bsb $\times$ HCM & 0.1736 & 10.66 & 0.10 & & LG6 \\
\hline & B493 x QAL & 0.1990 & 8.03 & 0.11 & $0.21^{\mathrm{Ns}}$ & LG6 \\
\hline Scar-AFLP3 x GGGCAT165-Q/H & Bsb $\times$ HCM & 0.1531 & 10.35 & 0.50 & & LG6 \\
\hline & B493 x QAL & 0.1108 & 20.52 & 0.36 & $0.86^{\mathrm{Ns}}$ & LG6 \\
\hline Scar-AFLP3 x GGTCTT350-4/H & Bsb $\times$ HCM & 0.1608 & 9.03 & 1.48 & & LG6 \\
\hline & B493 x QAL & 0.0808 & 19.12 & 1.40 & $2.88^{\mathrm{Ns}}$ & LG6 \\
\hline Scar-AFLP3 x GGGCAA289-Q/H & Bsb $\times$ HCM & 0.1144 & 12.55 & 0.16 & & LG6 \\
\hline & B493 x QAL & 0.0927 & 23.33 & 0.10 & $0.27 \mathrm{ss}$ & LG6 \\
\hline ACACAA319-4/H x GGGCAT165-Q/H & Bsb $\times$ HCM & 0.0667 & 20.16 & 4.03 & & LG6 \\
\hline & B493 x QAL & 0.2557 & 2.64 & 5.85 & $9.88^{* *}$ & LG6 \\
\hline ACACAA319-4/H x GGTCTT350-4/H & Bsb $\times$ HCM & 0.1573 & 10.17 & 0.03 & & LG6 \\
\hline & B493 x QAL & 0.1439 & 11.52 & 0.03 & $0.06^{\mathrm{Ns}}$ & LG6 \\
\hline ACACAA319-4/H x GGGCAA289-Q/H & Bsb $\times$ HCM & 0.1252 & 13.08 & 0.58 & & LG6 \\
\hline & B493 x QAL & 0.2139 & 3.21 & 1.12 & $1.70^{\mathrm{Ns}}$ & LG6 \\
\hline GGGCAT165-Q/H x GGTCTT350-4/H & Bsb $\times$ HCM & 0.0790 & 16.74 & 0.27 & & LG6 \\
\hline & B493 x QAL & 0.0000 & 5.92 & 2.65 & $2.95^{\text {Ns }}$ & LG6 \\
\hline GGGCAT165-Q/H x GGGCAA289-Q/H & Bsb $\times$ HCM & 0.0713 & 17.34 & 0.14 & & LG6 \\
\hline & B493 x QAL & 0.0555 & 29.74 & 0.09 & $0.24^{\mathrm{Ns}}$ & LG6 \\
\hline GGTCTT350-4/H x GGGCAA289-Q/H & Bsb $\times$ HCM & 0.0713 & 17.34 & 0.14 & & LG6 \\
\hline & B493 x QAL & 0.055 & 29.74 & 0.09 & 0.24 ss & LG6 \\
\hline
\end{tabular}

Ns,*,**Nonsignificant or significant at $p$ values of $1 \%$ or $5 \%$, respectively, by chi-square test. 
F $_{2}$ POPUlations. Twenty-eight conserved AFLP fragments were used to map the two populations. The amplicon size estimated in the individual population autoradiograms differed slightly, so confirmation of band identity and determination of band size was made when samples of each population were evaluated in the same acrylamide gel. A code representing both parents was used to represent a conserved AFLP fragment. For example: GGGCAA229-4/H marker represents a conserved AFLP identified in the primer combination GGGCAA, with an estimated fragment size of $229 \mathrm{bp}$ when both samples of both populations were run in the same acrylamide gel, where the origin of the fragment was designated $4=\mathrm{B} 493$ and $\mathrm{H}=\mathrm{HCM}$. B and Q were used to represent Brasilia and QAL parents in the marker name (Santos and Simon, 2002a).

AFLPbands of similar molecular size, amplified with the same primer combination in two different recombinant inbred lines of Arabidopsis were assumed to correspond to the same locus to compare position on the linkage groups without presenting any merged map groups (Alonso-Blanco et al., 1998). This is the first effort to apply sequence-conserved and shared AFLPs of the same size as anchors in order to merge linkage groups in carrot. Six merged linkage groups are presented in Fig. 1. The merged linkage groups from Brasilia x HCM and B493 x QAL were based on two codominant markers scored in both populations and in 28 conserved dominant AFLP markers. Heterogeneity tests were performed for all 30 conserved AFLPfragments originally mapped in both populations and two of them were dropped because they had chi-square values with $<0.01$ probability (Table 1 ). According to Stam and van Ooijen (1995), it is always a good practice to test the data for possible heterogeneity in the recombination fraction when the intent is to merge populations. Except for the linkage group 5, which did not have any codominant markers, the map merging incorporated the codominant markers presented in one group in the final merged group. It is not clear if merging linkage groups with predominantly dominant markers increased the repulsion phase problem or reduced the bias by incorporating rare codominant markers from one population into another.

The average marker spacing was $3.75 \mathrm{cM}$ (Fig. 1) in the six merged linkage groups while the average marker spacing was 4.8, 4.8, 5.5 and 5.1 cM in the Brasilia-, HCM-, B493 and QAL-coupling phase maps, respectively (Santos, 2001). Gaps up to $19.8,15.310 .9,10.4,9.6$, and $8.0 \mathrm{cM}$ were observed in the merged linkage groups 1, 2, 3, 4, 5, and 6, respectively (Fig.1). Similar gaps were also observed with the linkage coupling phase maps of the parents (Santos, 2001), indicating that linkage group integration did not double the bias, which comes with repulsion phases. Totaling up the length of coupling phase maps, the markers covered a total of $1188 \mathrm{cM}$ and $1114 \mathrm{cM}$ for all nine linkage groups in $\mathrm{F}_{2}$ progenies of Brasilia $\times$ HCM and B493 x QAL, respectively (Santos, 2001). The length of the six merged groups was $518 \mathrm{cM}$ while the length of the six merged coupling linkage maps were $795.3 \mathrm{cM}$ and 699.0 in $_{2}$ progenies of Brasilia $x \mathrm{HCM}$ and B493 x QAL, respectively. Staub et al., (2000) reported a reduction in total map distance when cucumber maps of four mapping populations were merged into only one.

Merged linkage groups incorporated approximately equal numbers of AFLP fragments from the four different parents, suggesting a reduction of the repulsion phase problem. The present merged groups should be seen not only as an initial effort to integrate different mapping populations of carrot based on conserved AFLP sequences but also as a starting point to generate better maps as more codominant markers are identified.
Merging linkage groups and maps based on common markers across the mapping populations represents an additional effort 1) to cross-validate mapping strategies and statistical approaches, 2) to identify QTL regions controlling economic traits within and among species, 3) to compare and to identify homologous maps among species and 4) to manage genomic information built into genome databases (Liu, 1997)

The CURRENT CARROT MAPPING EFFORT AND FUTURE PERSPECTIVES. The frequently severe inbreeding undergone by segregating generations of carrots (Peterson and Simon, 1986) has made difficult the development of recombinant inbred mapping populations to efficiently apply dominant markers. Molecular linkage maps of carrot $\mathrm{F}_{2}$ populations to date have been unable to defeat the barrier of repulsion linkage phases and the most extensive linkage map until now, published by Vivek and Simon (1999), is a simple coupling linkage phase for most linkage groups, due to the absence of enough codominant markers to efficiently order the mixed phases. Based on the results presented in the individual population maps and in the merged maps, it is reasonable to assume that at least three codominant markers per linkage group are necessary to successfully integrate mixed coupling and repulsion phases. Considering the low numbers of PCR based codominant markers among different carrot molecular studies, as reported in this study, it will be not a simple task to include at least three codominant markers per linkage group.

AFLP is a fast and reliable approach to generate a large number of polymorphic DNA markers. At least three scenarios could be pursued with carrot genomic mapping: 1) designing mapping populations that are not affected by the dominance, such as backcrosses, recombinant inbred lines or other inbred lines populations, 2) using updated analytical tools (such as AFLPQuantar Pro technology developed by Keygene Products B.V.; Wageningen, The Netherlands), that are able to quantify band intensity and provide codominant information of AFLP fingerprints and 3) converting conserved dominant AFLP to codominant markers and mapping these to specific targeted linkage groups by applying laborious and time consuming techniques, such as inverse PCR (Bradeen and Simon, 1998). Development of new mapping populations to efficiently map dominant markers may require new mapping efforts to incorporate previous maps with more markers to join the maps.

The extensive genome coverage in this study, however, is suitable to confidently apply QTL analysis and to perform powerful statistical analysis for at least for six merged groups. With this, for example, some progress in the identification of genes in the carotenoid pathway (Santos and Simon, 2002b) can be expected. Application of conserved mapping techniques as we describe here is of particular importance for these types of application since only a few biochemical pathways have been studied with the linkage analysis and QTL mapping. To identify not only collinear and conserved genomic regions within and among species, but also to explain the quantitative variation observed in the final products of a given pathway consensus mapping of unrelated populations will be of paramount importance.

\section{Literature Cited}

Alonso-Blanco, C., A.J.M. Peeters, M. Koornneef, C. Lister, C. Dean, N. van den Bosch, J. Pot, and T.R. Kuiper. 1998. Development of an AFLP based linkage map of Ler, Col and Cvi Arabidopsis thaliana ecotypes and construction of a Ler/Cvi recombinant inbred line population. Plant J. 14 (2):259-271.

Arumuganathan, K. and E.D. Earle. 1991. Nuclear DNA content of some 
important plant species. Plant Mol. Biol. Rptr. 9:208-218.

Boiteux, L.S. 2000. Characterization of the Meloidogyne javanica resistance locus employing molecular markers and isolation of candidate disease resistance loci in the carrot (Daucus carota L.) genome. Univ. Wis.-Madison PhD diss. abstr. DAI-B61/11 p. 5645.

Boiteux, L.S., M.E.N. Fonseca, and P.W. Simon. 1999. Effects of plant tissue and DNA purification method on randomly amplified polymorphic DNA-based genetic analysis in carrot. J. Amer. Soc. Hort. Sci. 124:32-38.

Bradeen, J.M. and P.W. Simon. 1998. Conversion of an AFLP fragment linked to the carrot $Y 2$ locus to a simple, codominant, PCR-based marker form. Theor. Appl. Genet. 97:960-967.

Burr, B., F.A. Burr, K.H. Thompson, M.C. Albertson, and C.W. Stuber. 1988. Gene mapping with recombinant inbreds in maize. Genetics 118:519-526.

Castiglioni, P., C Pozzi., M. Heun, V. Terzi, K.J. Muller, W. Rohde, and F.Salamini. 1998. An AFLP-based procedure for the efficient mapping of mutations and DNA probes in barley. Genetics 149:2039-2056.

Grattapaglia, D. and R.Sederoff. 1994. Genetic linkage maps of Eucalyptus grandis and Eucalyptus urophylla using a pseudo-testcross: Mapping strategy and RAP markers. Genetics 137:1121-1137.

Jiang, C. and Z.B Zeng. 1997. Mapping quantitative trait loci with dominant and missing markers in various crosses from two inbred lines. Genetica 101:47-58.

Keim, P., J.M. Schupp, S.E. Travis, K. Clayton, T.Zhu, L. Shi,A. Ferreira, and D.M. Webb. 1997. A high-density soybean genetic map based on AFLP markers. Crop Sci. 37:537-543.

Knapp, S.J., J.L. Hooloway, W.C. Bridges, and B.H. Liu. 1995. Mapping dominant markers using F matings. Theor. Appl. Genet. 91:74-81.

Kosambi, D.D. 1944. The estimation of map distances from recombination values. Ann. Eugen. 12:172-175.

Krutovskii, K.V., S.S. Vollmer, F.C Sorensen, W.T Adams, S.J. Knapp and S.H. Strauss. 1998. RAPD genome maps of douglas-fir. J. Hered. 89:197-205.

Lander, E.S., P. Green, J. Abrahamson, A. Barlow, M.J. Daly, S.E. Lincoln, and L. Newburg. 1987. MAPMAKER: An interactive computer package for constructing primary genetic linkage maps of experimental and natural populations. Genomics 1:174-181.

Liu, B.H. 1997. Statistical genomics: Linkage, mapping, and QTL analysis. CRC Press, Boca Raton, Fla.

Marques, C.M., J.A. Araujo, J.G. Ferreira, R Whetten., D.M. O’Malley, B.H. Liu and R. Sederoff. 1998. AFLP genetic maps of Eucalyptus globulus and E. tereticornis. Theor. Appl. Genet. 96:727-737.

Mather, K. 1936. Types of linkage data and their value. Ann. Eugen. 6:399-410.

Niemann, M., L. Westphal, and G. Wricke. 1997. Analysis of microsatellite markers in carrot (Daucus carota L. sativus). J. Appl. Genet. 38A:20-27.

Paran, I. and R. W. Michelmore. 1993. Development of reliable PCRbased markers linked to downy mildew resistance genes in lettuce. Theor. Appl. Genet. 85:985-993.

Peterson, C.E. and P.W. Simon. 1986. Carrot breeding, p. 321-356. In: M.J. Bassett (ed.). Breeding vegetable crops. AVI Publ. Co., Westport, Conn.
Plomion, C., B.H. Liu, and D.M. O'Malley. 1996. Genetic analysis using trans-dominant linked markers in an $\mathrm{F}_{2}$ family. Theor. Appl. Genet. 93:1083-1089.

Remington, D.L., R.W. Whetten, B.H. Liu, and D.M. O'Malley 1999. Construction of an AFLP genetic map with nearly complete genome coverage in Pinus taeda. Theor. Appl. Genet. 98:1279-1292.

Santos, C.A.F. 2001. Biometrical studies and quantitative trait loci associated with major products of the carotenoid pathway of carrot (Daucus carota L.). Univ. Wis.-Madison PhD diss. abstr. DAI-B02/07 p. 3080.

Santos, C.A.F. and P.W. Simon. 2002a. Some AFLP amplicons are highly conserved DNA sequences mapping to the same linkage groups in two F2 populations of carrot. Genet. Mol. Biol. 25:195-201.

Santos, C.A.F. and P.W. Simon. 2002b. QTL analyses reveal clustered loci for accumulation of major provitamin A carotenes and lycopene in carrot roots. Mol. Genet. Genom. 268:122-129.

Schafer-Pregl, R, D.C. Borchardt, E. Barzen, C. Glass, W. Mechelke, J.F. Seitzer, and F.Salamini. 1999. Localization of QTLs for tolerance to Cercospora beticola on sugar beet linkage groups. Theor. Appl. Genet. 99:829-836.

Schulz B., L. Westphal, and G. Wricke. 1994. Linkage groups of isozymes, RFLP and RAPD markers in carrot (Daucus carota L. sativus). Euphytica 74:67-76.

Sharma, A.K. and N.K. Bhattacharyya. 1954. Further investigations on several genera of Umbelliferae and their relationship. Genetica 30:1-68

Stam, P. and J.W.van Ooijen 1995. JOINMAP Version 2.0: Software for the calculation of genetic maps. CPRO-DLO, Wageningen, The Netherlands.

Staub, J.E., F.C. Serquen, and N. Katzir. 2000. Towards an integrated linkage map of cucumber: Map merging. Acta Hort. 510:357-366.

van Ooijen, J.W. 1994. A computer program for drawing genetic linkage maps. CPRO-DLO, Wageningen, The Netherlands.

Vivek, B.S.and P.W. Simon. 1999. Linkage relationships among molecular markers and storage root traits of carrot (Daucus carota L. spp. sativus). Theor. Appl. Genet. 99:58-64.

Vos, P., Hogers R.,M. Bleeker, M. Reijans, T. van der Lee, T. Hornes, A. Frijters, J. Pot, J. Peleman, M. Kupier, and M.Zabeau. 1995 AFLP:Anew technique for DNA fingerprinting. Nucl. Acids Res. 23:4407-4414.

Vuylsteke, M., R. Mank, R. Antonise, E. Bastiaans, M.L. Senior, C.W. Stuber, A.E.Melchinger, T. Lubberstedt, X.C. Xia, P. Stam, M. Zabeau, and M. Kuiper. 1999. Two high-density AFLP linkage maps of Zea mays L.: Analysis of distribution of AFLP markers. Theor. Appl. Genet. 99:921-935.

Westphal, L. and G. Wricke. 1989. Genetic analysis of DIA, GOT and PGI isozyme loci in Daucus carota L. ssp. sativus. Plant Breed. 102: 51-57.

Westphal, L. and G. Wricke. 1991. Genetic and linkage analysis of isozyme loci in Daucus carota L. Euphytica 56:259-267.

Westphal, L. and G. Wricke. 1997. Construction of a linkage map of Daucus carota L. sativus and its application for the mapping of disease resistance and restorer genes. J. Appl. Genet. 38A:13-19.

Young, W.P., J.M. Schupp, and P. Keim. 1999. DNA methylation and AFLP marker distribution in the soybean genome. Theor. Appl. Genet. 99:785-790. 